Journal of the Operations Research

Society of Japan

Vol. 33, No. 4, September 1990

\title{
RANDOMIZED DECISION STRATEGY FOR THE HIERARCHICAL OPTIMIZATION PROBLEMS
}

\author{
Mikio Kubo Hiroshi Kasugai \\ Waseda University
}

(Received October 20, 1989; Revised October 3, 1990)

\begin{abstract}
The hierarchical decision problem is a generic name of the problems whose common characteristics are:

1. They contain two or more levels of decision making,

2. Precise information about what will happen is not known a priori,

3. As the level is increased, more information can be available.

We introduce a new solution method refereed to as the randomized decision strategy for the hierarchical decision problems.

Two well-known and practical situations, which are the hierarchical scheduling problem and the hierarchical vehicle routing problem, are considered to illustrate this strategy.
\end{abstract}

\section{Introduction}

Several problems that we encounter in practical situations contain stochastic and combinatorial nature. 'Stochastic' means precise information about what will happen is not known a priori, and 'combinatorial' means the solution methods have to be enumerative.

Most operations research literatures are concentrated either on the deterministic combinatorial optimization problems assuming precious information is available, or on the stochastic linear programming problem ignoring combinatorial nature.

In many cases the deterministic combinatorial optimization algorithms can not be used because precious information can not be available a priori, and classical stochastic programming approaches (see for example [31] ) can not be used because the practical problems are not linear.

In order to bridge the gap between operation research practice and theory described above, we suggest a new technique for the solution of the problems that have combinatorial and stochastic nature. Many problems have sequence of decisions over time and hierarchical structure. So we concentrate on this type of the problem called the hierarchical decision problem (HDP). The hierarchical decision problem is a generic name of the problems whose common characteristics are:

1. They contain two or more levels of decision making,

2. Precise information about what will happen is not known a priori,

3. As the level is increased, more information can be available.

We introduce the randomized decision strategy for deriving practical algorithms to the HDPs. Roughly speaking, the randomized decision strategy is a class of randomized algorithms (see for example Welsh [41], Raghavan and Thompson [34], Raghavan [35]) whose 
common characteristic is to use randomness to overcome uncertainty. The general framework of this strategy is described in section 2. Two practical situations, which are the hierarchical scheduling problem and the hierarchical vehicle routing problem, are illustrated in section 3 and 4 , respectively.

2 General Framework of the Hierarchical Decision Problem and the Randomized Decision Strategy

Several practical problems in operations research can be formulated as the HDPs. Several approaches to these types of HDPs have been considered by several authors [5], [6], [14], [17], [23], [27].

We concentrate on the case where only two levels are recognized, and the decision problems occurring at each level are quite hard even when all data are known with certainty, i.e. they often belong to the class of NP-hard combinatorial optimization problems (see for example [19]), because many problems that we encounter in practice have such characteristics. The typical scenario can be described in the following way.

A decision maker must do the first level decision, which often called the aggregate level decision, using probabilistic information about the future events, here and now and the second level decision, which often called the detailed level decision, must be done after knowing a realization of random events. In other words, the tactical or strategic decision for the medium or long time horizon has to be determined at the first level; the operational decision for the short time horizon has to be determined at the second level

We cite some examples: the machine acquisition-job scheduling problem (the hierarchical scheduling problem) and the vehicle acquisition-routing problem (the hierarchical vehicle routing problem). In the job scheduling environment, the first level decision is the acquisition of the resources; the second level decision concerns about the allocation of resources to jobs. In the vehicle routing environment, the first level decision is the acquisition of the vehicles of several types; the second level decision concerns about the actual routes of the velicles acquired.

Our objective in this section is to suggest new technique, which we call the randomized decision strategy, for the solution of the general HDP. As a result, we can reduce the hierarchical decision problem to the easily solved one. This section is organized as follows. In subsection 2.1, we give a general framework of HDPs. In subsection 2.2, we review and unify the previous solution methods for the HDPs. In subsection 2.3, we introduce new solution strategy called the randomized decision strategy for the HDPs.

\subsection{Framework of the Hierarchical Decision Problem}

First, we give a general framework of the HDP.

The HDP can be formally defined as follows.

We denote the first level decision vector by $\xi_{1}$, the set of feasible decisions at the first level by $\Xi_{1}$ and the direct cost associated with $\xi_{1}$ by $f^{1}\left(\xi_{1}\right)$, where $f^{1}: \Xi_{1} \rightarrow \Re$ is a real function.

Probabilistic information of future events is represented by a vector $\tilde{\omega}$ that comes from a sample space $\Omega$ and has distribution function $F$. If we are given a realization $\omega$ of the random vector $\tilde{\omega}$ and the set of feasible decisions $\Xi_{2}\left(\xi_{1}, \omega\right)$ at the second level, then the expected cost $E\left[f^{2}\left(\xi_{1}, \tilde{\omega}\right)\right]$ of the second level can be represented as

$$
E\left[f^{2}\left(\xi_{1}, \tilde{\omega}\right)\right]=\int\left[\min _{\xi_{2} \in \Xi_{2}\left(\xi_{1}, \omega\right)} g\left(\xi_{1}, \xi_{2}, \omega\right)\right] d F(\omega)
$$


where $g: \Xi_{1} \times \Xi_{2} \times \Omega \rightarrow \Re$ is a real function that represents the cost associated with second level decision.

Then our objective is to determine the first level decision $\xi_{1} \in \Xi_{1}$ that minimizes

$$
f^{1}\left(\xi_{1}\right)+E\left[f^{2}\left(\xi_{1}, \tilde{\omega}\right)\right]
$$

In ordinary hierarchical planing models, the second level problem is an NP-hard combinatorial optimization problem. This means that finding an optimal solution of the second level problem in (1) is very hard task. Furthermore, the integrand in (1) is hard to evaluate because typical hierarchical problems have very large number of random variables.

Several classes of algorithms can be induced by approximation of the expected cost at the second level. We review them in the next subsection.

\subsection{Previous Works to the HDPs}

The previous works use the following approximation:

1. Monte Carlo simulation [6], [17]:

The expected cost at the second level is approximately evaluated by Monte Carlo Simulation. Several realizations of the random variables are randomly sampled from a sample space $\Omega$ and the expected cost of the second level problem is evaluated. This method is sometimes called random sampling method.

Birtan and Hax [6] studied a hierarchical planning system in which each level is modeled as a separate deterministic planning problem and the overall system qualities are evaluated by Monte Carlo simulation technique.

Ad hoc simulation method was used by Eilon et al. [17] for the solution of the traveling salesman facility location problem, i.e. the problem to find an optimal location for the service unit so that the expected distance traveled to satisfy the demand from customer with known probability distributions.

If the second level decision problem belongs to the class of NP-hard problems, this technique is hard to adapt because we have to solve many hard problems to evaluate the performance of the hierarchical system and to determine the first level decision.

2. Asymptotic analysis [14],[27]:

In this approach, $E\left[f^{2}\left(\xi_{1}, \tilde{\omega}\right)\right]$ is replaced with an estimate $E^{A}$ and the first level decision is determined optimally or heuristically, and a heuristic is used to solve the second level problem so that the solution value of the heuristic is asymptotically equal to the estimate $E^{A}$ in some probabilistic sense.

Lenstra et al. [27] and Dempster et al. [14] proposed analytical evaluation procedures for the two-level hierarchical planning models. They modeled several practical problems such as the vehicle routing and scheduling problems. Their algorithms are based on the asymptotically optimal heuristics such as a partitioning heuristic for the vehicle routing problem and list scheduling heuristics for the scheduling problem.

3. Restriction of the solution space [5],[23]:

In this approach, the second level decision is restricted so that the expected cost of the second level can be easily evaluated. More precisely, we replace $\Xi_{2}\left(\xi_{1}, \omega\right)$ with the set $\breve{\Xi}_{2}\left(\xi_{1}, \omega\right)$ of the restricted feasible solutions at the second level so that the expectation

$$
E\left[\breve{f}^{2}\left(\xi_{1}, \tilde{\omega}\right)\right]=\int\left[\min _{\xi_{2} \in \check{\Xi}_{2}\left(\xi_{1}, \omega\right)} g\left(\xi_{1}, \xi_{2}, \omega\right)\right] d F(\omega)
$$


can be computed easily.

This method has been used for the solution of the traveling salesman facility location problem (TSFLP) in which the first level decision is to determine the location point of the vehicle knowing only probabilistic information about the customer demands; the second level decision is to determine the route of the vehicle after knowing the actual customer demands. Burness and White [8] and Berman and Simchi-Levi [3] proposed approximate algorithms in which the second level problem, the traveling salesman problem, is approximated by direct distances from node 1 . The worst case bound of the latter algorithm is improved by Bertsimas [5]. Among several heuristics a priori strategy is most valuable. The idea of using an a priori tour was first proposed by Jaillet [23] for the probabilistic version of the traveling salesman problem and extended by Bertsimas [5] for other combinatorial optimization problems. Berman and Simchi-Levi [4] and Bertsimas [5] used the a priori tour strategy for the TSFLP, i.e. a service unit visits the nodes at a particular instance in the same order as they appear in the a priori (pre-determined) tour, instead of using the optimal traveling salesman tour at every instances.

\subsection{General Outline of Randomized Decision Strategy and Relation to Other Strategies}

Several researchers considered the restriction method of the solution space that we reviewed in the previous subsection. Instead of restricting the solution space, we relax it, i.e. the second level decision is relaxed so that the expected cost at the second level can be easily evaluated. More precisely, we replace $\Xi_{2}\left(\xi_{1}, \omega\right)$ with the set $\tilde{\Xi}_{2}\left(\xi_{1}, \omega\right)$ of the relaxed feasible solutions so that the expectation $E\left[f^{2}\left(\xi_{1}, \tilde{\omega}\right)\right]$ in (2) can be computed easily.

Among several relaxation methods of the solution, we adopt randomized relaxation in which the second level decision is replaced with the randomized one so that the expected cost over the infinite time horizon is minimized. We call this technique the randomized decision strategy in the sequel.

The randomized decision strategy is known to the mixed strategy in game theory (see for example [25]). In game theoretical terms, the HDP can be seen as a three stage game in which only two players are recognized; one is a decision maker (player 1), another is probabilistic future events (player 2). The rule of the game is as follows (see Figure 1):

First, player 1 determines the first decision from a finite set $\Xi_{1}$ of the feasible decisions. Then player 2 uses the mixed strategy, i.e. the decision is determined by a coin tossing (randomly), at the second stage. At the third stage, player 1 determines the recourse decision via solving combinatorial optimization problems, and evaluates the expected solution value. Game value is the expectation of all possible solution values at the third stage.

Our strategy is to use the mixed strategy instead of determining an optimal solution at the third stage Then the evaluation of expectation is much easier; we can determine the first level decision by evaluating the expected value of two mixed strategies at the second and third stages (see Figure 2).

Derman and Klein [15] considered the Markov decision strategy for the traveling salesman problem with frequency constraints. In this contexts, the randomized decision strategy can be seen as the Markov decision strategy in which we determine transient probability so that the expected cost over the infinite time horizon is minimized.

The objective of the randomized decision strategy is twofold:

1. deriving a lower bound of the second level decision problem, 


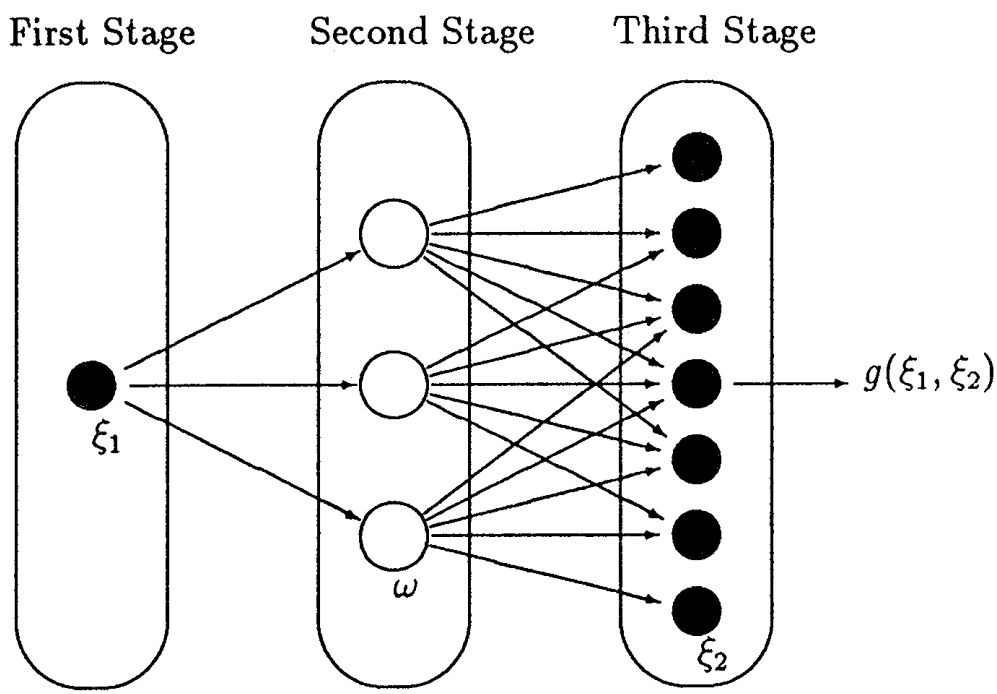

- Decision Stage

$\bigcirc \quad$ : Probabilistic Stage

Figure 1: Three Stage Game : The level hierarchical Decision can be represented as the three stage game.

\section{First Stage Mixed Strategy}

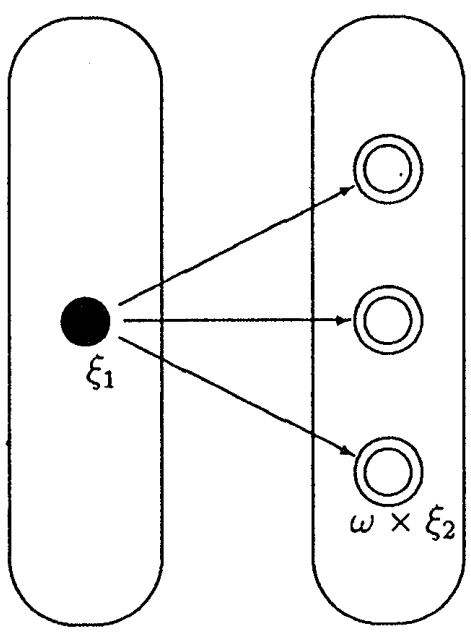

- : Decision Stage

(a) : Combination of Two Probabilistic Stages

Figure 2: Randomized (Mixed) Strategy : The second and third stages of the original three stage game are combined into the mixed stage. 
2. giving a feasible solution (an upper bound) with a probabilistic guarantee.

$<$ Lower Bound >

Since we relax the second level decision by the randomized one, we can get the following relation:

$$
\Xi_{2}\left(\xi_{1}, \omega\right) \subseteq \tilde{\Xi}_{2}\left(\xi_{1}, \omega\right)
$$

This indicates that the straightfoward application of the randomized decision strategy gives the lower bound of the second level decision problem.

\section{$<$ Upper Bound >}

Since we relax the set of feasible solution in the randomized decision strategy, the solution obtained may not be feasible with high probability. We avoid this using the chance constraint method (see Charnes and Cooper [11]). Given a probability $0<\epsilon<1$ that is determined by a decision maker, we modify the second level problem to give the feasible solution at least with probability $1-\epsilon$. If the decision maker sets $\epsilon$ small, the solution obtained by the randomized decision strategy is feasible with high probability, while if the decision maker sets $\epsilon$ large, the solution obtained by the randomized decision strategy is infeasible with high probability.

If we use randomized decision strategy $\xi_{2}$ that is drawn from a sample space $\tilde{\Xi}_{2}\left(\xi_{1}, \omega\right)$ and denote its distribution function by $G$, then the expected cost $E\left[f^{2}\left(\xi_{1}, \tilde{\omega}\right)\right]$ at the second level can be approximated as follows:

$$
\begin{aligned}
E\left[\tilde{f}^{2}\left(\xi_{1}, \tilde{\omega}\right)\right] & =\int g\left(\xi_{1}, \tilde{\xi}_{2}, \omega\right) d F(\omega) \\
& =\iint g\left(\xi_{1}, \xi_{2}, \omega\right) d F(\omega) d G\left(\xi_{2}\right) .
\end{aligned}
$$

Then our objective is to determine first level decision $\xi_{1} \in \Xi_{1}$ and second level randomized decision $\tilde{\xi}_{2}$ simultaneously, that minimize

$$
f^{1}\left(\xi_{1}\right)+E\left[\tilde{f}^{2}\left(\xi_{1}, \tilde{\omega}\right)\right]
$$

subject to

$$
\begin{gathered}
\operatorname{Pr}\left\{\tilde{\xi}_{2} \in \Xi_{2}\left(\xi_{1}\right)\right\} \geq 1-\epsilon, \\
\xi_{1} \in \Xi_{1} .
\end{gathered}
$$

This problem looks more difficult than the original one. But in several hierarchical planning models, this problem can be replaced with more simply structured one as shown in section 3 and section 4 .

\subsection{How to Use the Randomized Decision Strategy}

Relation between several strategies is summarized in Figure 3.

In practice, the following combined strategy is useful to solve hierarchical planning problems systematically.

1. Use asymptotic analysis as a first cut of the problem.

2. Use the randomized decision strategy or a priori strategy to get more information of the problem. 


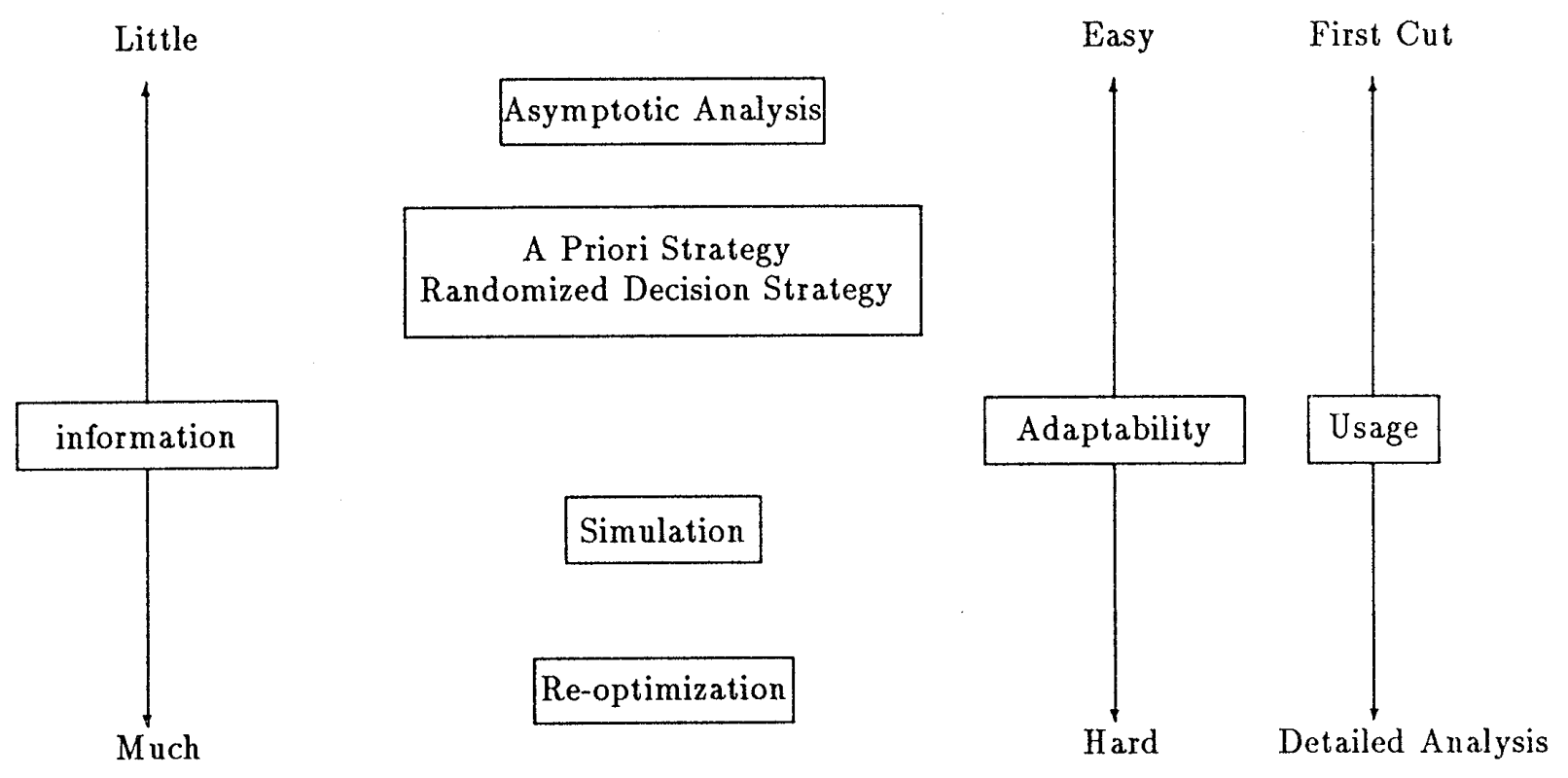

Figure 3: Relation between Strategies

3. Use simulation analysis or the re-optimization strategy to analyze the problem in detail.

We conjecture that the randomized decision strategy is another useful tool for probabilistic and hierarchical combinatorial optimization problems. To illustrate the randomized decision strategy, we will present a hierarchical scheduling problem and a hierarchical vehicle routing problem in the subsequent sections.

\section{The Hierarchical Scheduling Problem}

In this section, we consider a typical hierarchical planning scenario in the practical scheduling environment called the hierarchical scheduling problem (HSP) that can be defined as follows:

Let $M=\{1,2, \cdots, m\}$ be the set of machines and $J=\{1,2, \cdots, n\}$ the set of jobs. Each job $j \in J$ has random processing time $\tilde{p}_{j}$ that has distribution function $F$. If job $j$ is processed at machine $i$, the processing cost $d_{i j}$ is incurred. Each machine $i \in M$ has the acquisition cost $c_{i}$. If the sum of processing time assigned to machine $i$ is more than $q_{i}$, the overtime penalty cost is incurred. At the first (aggregate) level, one must decide on the acquisition of several types of machines. At the second (detailed) level, one must decide on the assignment of jobs to the machines acquired at the first level. Our objective is to minimize the sum of the total acquisition cost, the processing cost, and the overtime penalty.

Dempster et al. [14] considered the simple hierarchical scheduling problem; they assume that machines are identical or uniform. The second level problem is solved by a list scheduling heuristic, while the first level problem is solved by a simple formula or a greedy heuristic. Under several assumptions, they prove that their combination algorithms of several heuristics give asymptotically optimal solutions.

We may assume more general scheduling environment. But for comparison, our model is similar to theirs. Remark that our solution strategy is more general, so we can extend the result developed in this section to more practical and realistic cases. 
This section is organized as follows. In subsection 3.1, we explain precious definition of our model. In subsection 3.2, an approximate solution method based on the randomized decision strategy is presented.

\subsection{Problem Definition of the HSP}

The HSP has two level decision stages; aggregate level and detailed level. At the aggregate level, we must decide on the acquisition of several types of machines. More precisely, the problem can be stated as follows:

\section{(ALP : Aggregate Level Problem)}

$$
\min \sum_{i \in M} c_{i} y_{i}+\int g(y, p) d F(p)
$$

subject to

$$
y_{i} \in\{0,1\} \quad \forall i \in M .
$$

Given a realization $p=\left(p_{1}, p_{2}, \cdots, p_{n}\right)$ of random vector $\tilde{p}$ and the first level decision vector $y=\left(y_{1}, y_{2}, \cdots, y_{m}\right)$, we can calculate the second level cost function $g(y, p)$ as follows.

$$
g(y, p)= \begin{cases}z^{*}(y, p) & \text { if } z^{*}(y, \Phi) \neq \infty \\ \text { penalty of overtime } & \text { if } z^{*}(y, \Phi)=\infty\end{cases}
$$

where $z^{*}(y, p)$ is an optimal solution of the following second level decision problem:

(DLP : Detailed Level Problem)

$$
z^{*}(y, p)=\min \sum_{i \in M^{\prime}} \sum_{j \in J} d_{i j} x_{i j}
$$

subject to

$$
\begin{gathered}
\sum_{i \in M^{\prime}} x_{i j}=1 \quad \forall j \in J, \\
\sum_{j \in J} p_{j} x_{i j} \leq q_{i} \quad \forall i \in M^{\prime}, \\
x_{i j} \in\{0,1\} \quad \forall i \in M^{\prime}, \forall j \in J,
\end{gathered}
$$

where $M^{\prime}=\left\{i \in M \mid y_{i}=1\right\}$.

\subsection{Randomized Algorithm for the HSP}

In this subsection, we use the randomized decision strategy to get an approximate solution method to the HSP. The reason we must use the approximation method is threefold:

1. The overtime penalty cost is difficult to estimate.

2. Since the number of the possible instances is exponentially large, the integrand in (9) is hard to estimate.

3. The subproblem DLP is NP-complete; we can not solve the DLP many times. 
The penalty of overload can be incorporated using the chance constraint method (see for example Charnes and Cooper [11]). Instead of minimizing the penalty function $g(y, \Phi)$ in (11), we determine the first level decision so that the second level problem has a feasible solution with probability at least $1-\epsilon$. Furthermore, the second level decision is relaxed to the randomized one; the second level decision vector $\tilde{x}$ is random variable drawn from distribution $G$. As a result, we get the following randomized decision problem:

(Randomized Decision Problem)

$$
\min \sum_{i \in M} c_{i} y_{i}+\int \sum_{i \in M} \sum_{j \in J} d_{i j} x_{i j} d G(x)
$$

subject to

$$
\begin{gathered}
\operatorname{Pr}\left(\sum_{j \in J} \tilde{p}_{j} \tilde{x}_{i j} \leq q_{i} y_{i}, \forall i \in M\right) \geq 1-\epsilon, \\
y_{i} \in\{0,1\} \quad \forall i \in M .
\end{gathered}
$$

If we assume randomized decision $\tilde{x}_{i j}$ at the second level and the processing time $\tilde{p}_{j}$ are independent random variables such that $0 \leq \tilde{x}_{i j} \leq 1$ and $0 \leq \tilde{p}_{j} \leq 1$, the problem above can be approximated by the following problem:

(Reduced Randomized Decision Problem)

$$
\min \sum_{i \in M} c_{i} y_{i}+\sum_{i \in M} \sum_{j \in J} E\left(\tilde{d}_{i j}\right) E\left(\tilde{x}_{i j}\right)
$$

subject to

$$
\begin{gathered}
\sum_{i \in M} E\left(\tilde{x}_{i j}\right)=1 \quad \forall j \in J, \\
\sum_{j \in J} E\left(\tilde{p}_{j}\right) E\left(\tilde{x}_{i j}\right) \leq \hat{q}_{i} y_{i} \quad \forall i \in M, \\
y_{i} \in\{0,1\} \quad \forall i \in M,
\end{gathered}
$$

where $\hat{q}_{i}$ is determined as follows:

$$
\hat{q}_{i}=q_{i}+\frac{3}{2} \gamma-\sqrt{3 q_{i} \gamma+\frac{9}{4} \gamma^{2}}
$$

and

$$
\gamma=\ln \frac{m}{\epsilon}
$$

The problem defined above is essentially the capacitated location problem that has been extensively studied by many authors (see for survey [28]), and can be solved efficiently though the problem is known to be NP-hard [19].

Let the optimal solution vectors of the problem above be $\bar{y}$ and $\bar{x}$. The first level decision is deterministic; if $\bar{y}_{i}=1$ then machine $i$ must be acquired, while the second level decision is the randomized one; job $j$ must be assigned to machine $i$ with probability $\bar{x}_{i j}$.

When the deterministic information of jobs is available, we may re-optimize the second level decision problem. In this case, the second stage problem is essentially the parallel shop scheduling problem. We may assign jobs to the machines acquired at the first stage by the randomized decision strategy. This strategy gives an approximate solution of the second stage decision problem, but the solution is guaranteed to be feasible with high probability. We use the following lemma to analyze the worst case bound of our algorithms. 
Lemma 1 (Hoeffding [22], see also Angluin and Valiant [2], Raghavan [35] ) If $\Psi_{1}, \Psi_{2}, \cdots, \Psi_{N}$, are independent random variables such that $0 \leq \Psi_{i} \leq 1$ for $i=1,2, \cdots, N$. For $0<\beta<1$

$$
\operatorname{Pr}(\Psi \geq(1+\beta) S)<\exp ^{-\frac{\beta^{2} S}{3}}
$$

where

$$
S=\sum_{k=1}^{N} E\left(\Psi_{k}\right)
$$

and

$$
\Psi=\Psi_{1}+\Psi_{2}+\cdots+\Psi_{N}
$$

To get the worst case performance of randomized decision strategy for HSP, we need scaling of random variables $\tilde{d}_{i j}$. Without loss of generality, we can scale the problem instance so that the random variables $\tilde{d}_{i j}$ for all $i \in M, j \in J$ are less or equal to 1 . If we adapt the randomized decision strategy after appropriate scaling of variables, we can get the worst case performance in a probabilistic sense as shown in the following theorem.

Theorem 1 Let $z_{1}=\sum_{i \in M} c_{i} \bar{y}_{i}$ and $z_{2}=\sum_{i \in M} \sum_{j \in J} E\left(\tilde{d}_{i j}\right) \bar{x}_{i j}$ where $\bar{y}$ and $\bar{x}$ are the optimal solution vectors of the reduced randomized decision problem (19)-(22). Assume that for $0<\epsilon<1$ and for all $k=1, \cdots, m$,

$$
\hat{q}_{k}>3 \ln \frac{m}{\epsilon}
$$

and

$$
z_{2}>3 \ln \frac{1}{\epsilon}
$$

Then the randomized decision strategy gives a feasible solution whose objective function value is less than $z_{1}+z_{2}+\sqrt{3 z_{2} \ln \frac{1}{\epsilon}}$ with probability at least $1-\epsilon$.

Proof: Consider a machine $j$. Observe that $\sum_{j \in J} \tilde{p}_{j} \tilde{x}_{i j}$ is the sum of random independent variables, we can adapt Lemma 1 with $\beta$ in $(25)$ being $\sqrt{\frac{3}{\hat{q}_{k}} \ln \frac{m}{\epsilon}}$. Since $\hat{q}_{j}>3 \ln \frac{m}{\epsilon}$, we get $\beta<1$. Since the expected value of the sum of the random variables is less or equal to $\hat{q}_{i}$, we can get

$$
\operatorname{Pr}\left(\sum_{j \in J} \tilde{p}_{i} \tilde{x}_{i j} \geq(1+\beta) \hat{q}_{i}\right) \leq \exp ^{-\beta^{2} \dot{q}_{i} / 3}
$$

This inequality means the probability that overload occurs is less or equal to $\frac{\epsilon}{m}=\exp ^{-\beta^{2} \hat{q}_{i} / 3}$. Consequently, we can get the probability with which all machines satisfy the production time constraints is at least $\left(1-\frac{\epsilon}{m}\right)^{m} \geq 1-\epsilon$.

Next we turn to prove the performance of the objective function value obtained by randomized decision strategy. We determine binary variables $x_{i}$ for all $i \in M$ by coin tossing. Since the trials of coin tossing are independent and $\tilde{d}_{i j} \bar{x}_{i j}$ is less or equal to 1 , we can adapt Lemma 1 with $\beta$ in (25) being $\sqrt{\frac{3}{z_{2}} \ln \frac{1}{\epsilon}}$. Remark that the assumption $z_{2}>3 \ln \frac{1}{\epsilon}$ means $\beta<1$. Thus we can get

$$
\operatorname{Pr}\left(\sum_{i \in M} \sum_{j \in J} \tilde{d}_{i j} \bar{x}_{i j} \geq(1+\beta) z_{2}\right) \leq \exp ^{-\beta^{2} z_{2} / 3}(=\epsilon) .
$$


This inequality shows the objective function value obtained by randomization is less than $z_{1}+(1+\beta) z_{2}=z_{1}+z_{2}+\sqrt{3 z_{2} \ln \frac{1}{\epsilon}}$ with probability at least $1-\epsilon$. This proves the theorem. I

We illustrated the randomized decision strategy using a simple scheduling problem. Our result can be easily extend to more practical variations of the hierarchical scheduling problem. Dempster et al. [14] described several extensions of the hierarchical scheduling problem such as the objective is to minimize the total or maximum tardiness when jobs have common due dates. Our algorithm is more flexible. We can extend our model to the case when jobs have different due dates or the general job shop case.

\section{The Hierarchical Vehicle Routing Problem}

In this section we consider a hierarchical vehicle routing problem (HVRP). This problem arises when we do not have exact information about the demands of customers who are served by vehicles of limited capacity from a common depot.

The HVRP is composed of two decision levels: at the first level, decision to acquire several types of vehicles has to be made based on imperfect information about the actual demands of future customers, and at the second level, the route of each vehicle has to be determined to satisfy the demands of all customers. Our objective is to minimize the sum of the acquisition costs of vehicles and the sum of the variable costs of the routes. Of course, decision at the second level must be made after the exact locations and demands of the customers are known.

We adapt the randomized decision strategy to the HVRP. Since the HVRP is more complex than the hierarchical scheduling problem, we must use several heuristics to develop an approximate algorithm for the HVRP.

This section is organized as follows. In subsection 4.1, we briefly review the previous works for the HVRPs. In subsection 4.2, precious definition of the HVRP which we concentrate on is described. In subsection 4.3, the randomized decision strategy combined with several heuristics is used to the HVRP. Analysis of the heuristic is also presented.

\subsection{Previous Works to the HVRP}

The HVRP is an extended version of the classical vehicle routing problem (VRP), in which customers have to be served by vehicles subject to constraints on the total load in each vehicle under deterministic information about customer locations and demands. The VRP has been treated by many researchers. Bodin et al. [7] provides an overview and Christofides et al. [12] suggested a general framework for the VRP.

Spaccamela, Rinnooy Kan and Stougie [37] considered a simplified version of the HVRP that can be defined as the following two stage decision problem. At the first level, the decision must be made about the number of vehicles acquired at a known identical cost to serve customers from a single depot. They assume the customers are uniformly distributed in a circle with the depot at its center. At the second level, the vehicles whose number must be decided on at the aggregate level must be routed to service customers to minimize the maximum route length assigned to a vehicle. They proposed a combination procedure of several heuristics and proved the asymptotic optimality of the algorithm.

Several authors considered a stochastic version of the VRP in which the demand of each customer is a random variable whose value is unknown until the vehicle arrives that customer. The objective is to minimize the expected daily costs that consists of the expected routing cost and the expected cost of servicing customers who could not be serviced. Dror and 
Trudeau [16] proposed a heuristic algorithm based on the Clark and Wright saving method [13].

Jaillet and Odoni [24] considered the VRP with demands generated according to Binomial distribution. The problem is to determine a fixed set of routes of minimal expected total length, which corresponds to the expected total length of the fixed set of routes plus the expected value of the extra distance that might be required by a particular realization of the random variables. They proposed two strategies and analyzed them.

\subsection{Problem Definition of the HVRP}

To provide a precious statement of this problem, we introduce several notations.

Let

$m$ be the number of vehicles,

$M$ be $\{1,2, \cdots, m\}$,

0 be the depot,

$V_{0}$ be the customer locations,

$V$ be $\{0\} \cup V_{0}$, i.e. $V$ be a set of nodes that are composed of customers plus the depot,

$c_{k}(k \in M)$ be the acquisition cost of vehicle $k$,

$q_{k}(k \in M)$ be the capacity of vehicle $k$,

$\tilde{\Phi}_{i}\left(i \in V_{0}\right)$ be the random variable that represents the demand of customer $i$,

$F$ be the probability density function of the random variable vector $\tilde{\Phi}=\left(\tilde{\Phi}_{1}, \cdots, \tilde{\Phi}_{\left|V_{0}\right|}\right)$,

$\Phi_{i}\left(i \in V_{0}\right)$ be the realized demand of customer $i$,

$d_{i j}(i \in V, j \in V-\{i\})$ be the direct travel cost from $i$ to $j$,

$z_{1}^{*}$ be the optimal value of the first level decision problem,

$z_{2}^{*}(y, \Phi)$ be the optimal value of the second level decision problem, which is the function of the first level decision vector $y=\left(y_{1}, \cdots, y_{m}\right)$ and the realized demand vector $\Phi=\left(\Phi_{1}, \cdots, \Phi_{\left|V_{0}\right|}\right)$,

$g(y, \Phi)$ be the recourse function of the second level problem.

Without loss of generality, we assume $0 \leq \tilde{\Phi}_{i} \leq 1$ for all $i \in V_{0}$.

Using the notations above, the HVRP can be formulated as the following two stage stochastic integer programming problem:

(First Level Decision Problem)

$$
z_{1}^{*}=\min \sum_{k \in M} c_{k} y_{k}+\int g(y, \Phi) d F(\Phi)
$$

subject to

$$
y_{k} \in\{0,1\} \quad \forall k \in M
$$


where

$$
y_{k}= \begin{cases}1 & \text { if vehicle } k \text { is acquired } \\ 0 & \text { otherwise }\end{cases}
$$

and

$$
g(y, \Phi)= \begin{cases}z_{2}^{*}(y, \Phi) & \text { if } z_{2}^{*}(y, \Phi) \neq \infty \\ \text { penalty of overload } & \text { if } z_{2}^{*}(y, \Phi)=\infty\end{cases}
$$

where function $z_{2}^{*}(y, \Phi)$ represents the optimal value of the second level decision problem given the first level decision vector $y$ and the realization of random vector $\Phi$.

More precisely $z_{2}^{*}(y, \Phi)$ is determined in the following way: Given a realization vector $\Phi$ of random variables and the first level decision vector $y$, the second level decision problem can be defined as follows.

\section{(Second Level Decision Problem)}

$$
z_{2}^{*}(y, \Phi)=\min \sum_{i \in V} \sum_{j \in V} d_{i j} \sum_{k \in M} \xi_{i j k}
$$

subject to

$$
\begin{gathered}
\sum_{j \in V} \sum_{k \in M} \xi_{i j k}=1 \quad \forall i \in V_{0}, \\
\sum_{j \in V} \xi_{i j k}=\sum_{l \in V} \xi_{l i k} \quad \forall i \in V, k \in M, \\
\sum_{i \in S} \sum_{j \in S} \xi_{i j k} \leq|S|-1 \quad \forall S \subseteq V_{0}, k \in M \\
\sum_{i \in V} \Phi_{i} \sum_{j \in V} \xi_{i j k} \leq q_{k} y_{k} \quad \forall k \in M, \\
\xi_{i j k} \in\{0,1\} \quad \forall i, j \in V, k \in M,
\end{gathered}
$$

where

(42) $\quad \xi_{i j k}= \begin{cases}1 & \text { if vehicle } k \text { visits node } j \text { immediately after visiting node } i \\ 0 & \text { otherwise. }\end{cases}$

The problem defined above is very hard one, because it has a stochastic nature and contains NP-hard problems. Thus, some approximation techniques must be used. In the next subsection, we develop an approximate algorithm based on the randomized decision strategy. 


\subsection{Randomized Algorithm for the HVRP}

We use three approximation methods that are

1. Replace the routing costs with the insertion costs to the seed customers,

2. The overload penalty costs are approximated by chance constraints, i.e. the probability with which the overload occurs is at least, say $\epsilon$,

3. Replace the deterministic decision with the randomized one.

The second level problem is essentially a vehicle routing problem (VRP) under deterministic information. Since routing decisions are very complex and we are not interested in the actual routes at the first level, we decomposed the second level decision problem into two decision problems, i.e. the vehicle allocation stage and the traveling salesman stage, and approximate the traveling salesman problem ( see Fisher and Jaikumar [18]).

The second stage problem can be rewritten in the following way:

(Second Level Decision Problem)

$$
z_{2}^{*}(y, \Phi)=\min \sum_{k \in M} f_{k}(x)
$$

subject to

$$
\begin{gathered}
\sum_{k \in V} x_{i k}=1 \quad \forall i \in V_{0}, \\
\sum_{i \in V} \Phi_{i} x_{i k} \leq q_{k} y_{k} \quad \forall k \in M, \\
x_{i k} \in\{0,1\} \quad \forall i \in V, k \in M,
\end{gathered}
$$

where

$$
x_{i k}= \begin{cases}1 & \text { if vehicle } k \text { is assigned to customer } i \\ 0 & \text { otherwise }\end{cases}
$$

and $f_{k}(x)$ is the optimal traveling salesman tour in $N_{k}=\left\{i \mid x_{i k}=1\right\}$, i.e. the set of customers who are assigned to vehicle $k$. Since $f_{k}(x)$ is a very complex nonlinear function, we use the following linear approximation:

$$
f_{k}(x)=\sum_{i \in V_{0}} \Delta_{i k} x_{i k}
$$

To construct a linear approximation of $f_{k}(x)$, we begin with a set $S$ of seed customers that are assigned to respective vehicles. The coefficient $\Delta_{i k}$ is then set equal to the cost of inserting customer $j$ into the route in which vehicle $k$ travels from the depot to customer $i_{k}$ and back directly, i.e.

$$
\Delta_{i k}=\min _{\ell \in S}\left\{d_{0 i}+d_{i \ell}-d_{0 \ell}, d_{i 0}+d_{\ell i}-d_{\ell 0}\right\}
$$

We replace the second level decision with the randomized one so that the expected cost over infinite time horizon is minimized. In this case, the decision variable $\tilde{x}_{i k}$ represents the probability with which vehicle $k$ is assigned to customer $i$. 
The penalty of overload can be incorporated using the chance constraint method (see for example Charnes and Cooper [11]). Instead of minimizing the penalty function $g(y, \Phi)$ in (35), we determine the first level decision so that the second level problem has a feasible solution with probability at least $1-\epsilon$.

If we are given randomized decision vector $\tilde{x}$ whose distribution function is $G$, then we can get the following stochastic programming problem.

(Randomized Decision Problem)

$$
\min \sum_{k \in M} c_{k} y_{k}+\int \sum_{i \in V_{0}} \sum_{k \in M} \Delta_{i k} x_{i k} d G(x)
$$

subject to

$$
\begin{gathered}
\operatorname{Pr}\left\{\sum_{i \in V} \tilde{\Phi}_{i} \tilde{x}_{i k} \leq q_{k} y_{k}, \forall k \in M\right\} \geq 1-\epsilon, \\
y_{k} \in\{0,1\} \quad \forall k \in M .
\end{gathered}
$$

Since the randomized decision problem defined above is too complex to solve directly, we use some approximation techniques. If we assume randomized decision variables $\tilde{x}_{i j}$ and the demand of each customer $\tilde{\Phi}_{i}$ are independent random variables such that $0 \leq \tilde{x}_{i j} \leq 1$ and $0 \leq \tilde{\Phi}_{i} \leq 1$, the randomized decision problem above can be approximated by the following mixed integer programming problem.

(Reduced Randomized Decision Problem)

$$
\bar{z}=\min \sum_{k \in M} c_{k} y_{k}+\sum_{i \in V_{0}} \sum_{k \in M} \Delta_{i k} E\left(\tilde{x}_{i k}\right)
$$

subject to

$$
\begin{gathered}
\sum_{k \in V} E\left(\tilde{x}_{i k}\right)=1 \quad \forall i \in V_{0}, \\
\sum_{i \in V} E\left(\tilde{\Phi}_{i}\right) E\left(\tilde{x}_{i k}\right) \leq \hat{q}_{k} y_{k} \quad \forall k \in M, \\
y_{k} \in\{0,1\} \quad \forall k \in M, \\
0 \leq E\left(\tilde{x}_{i k}\right) \leq 1 \quad \forall i \in V, k \in M,
\end{gathered}
$$

where

$$
\hat{q}_{k}=q_{k}+\frac{3}{2} \gamma-\sqrt{3 q_{k} \gamma+\frac{9}{4} \gamma^{2}}
$$

and

$$
\gamma=\ln \frac{m}{\epsilon} .
$$

The problem defined above is essentially the capacitated location problem that has been extensively studied by many authors (see for survey [28]), and can be solved efficiently though the problem is known to be NP-hard [19]. 
Let the optimal solution vectors of the problem above be $\bar{y}$ and $\bar{x}$. First level decision is deterministic; if $\bar{y}_{k}=1$ then vehicle $k$ must be acquired, while the second level decision is the randomized one; vehicle $k$ must be assigned to customer $i$ with probability $\bar{x}_{i k}$.

When deterministic information of customer demands is available, we may re-optimize the second stage decision problem. In this case, the second stage problem is essentially the vehicle routing problem.

We may assign vehicles acquired at the first stage to customers by the randomized decision strategy and solve the traveling salesman problem on the node set assigned to each vehicle. This strategy gives an approximate solution of the second stage decision problem, but the solution is guaranteed to be feasible with high probability. More formally, the performance of the randomized algorithm can be guaranteed by the following theorem.

Theorem 2 Given an $m$ disjoint partition $V_{1}, V_{2}, \cdots, V_{m}$ of $V$ that satisfies

$$
\sum_{i \in V_{k}} E\left(\tilde{\Psi}_{i}\right) E\left(\tilde{x}_{i k}\right) \leq \hat{q}_{k} \quad k=1, \cdots, m
$$

Assume that for $0<\epsilon<1$ and for all $k=1, \cdots, m$,

$$
\hat{q}_{k}>3 \ln \frac{m}{\epsilon}
$$

the total demand does not exceed the capacity of every vehicle $q_{k}=\hat{q}_{k}+\sqrt{3 \hat{q}_{k} \ln \frac{m}{\epsilon}}$ with probability at least $1-\epsilon$.

Proof: Consider a vehicle $k$. Observe that $\sum_{i \in V_{k}} \tilde{\Psi}_{i} \tilde{x}_{i k}$ is the sum of random independent variables, we can adapt Lemma 1 with $\beta$ in $(25)$ being $\sqrt{\frac{3}{\hat{q}_{k}} \ln \frac{m}{\epsilon}}$. Since $\hat{q}_{k}>3 \ln \frac{m}{\epsilon}$, we get $\beta<1$. Since the expected sum $\sum_{i \in V_{k}} E\left(\tilde{\Psi}_{i}\right) E\left(\tilde{x}_{i k}\right)$ is less or equal to $\hat{q}_{k}$, we can get

$$
\operatorname{Pr}\left\{\sum_{i \in V} \tilde{\Phi}_{i} \tilde{x}_{i k} \geq(1+\beta) \hat{q}_{k}\right\} \leq \exp ^{-\frac{\beta^{2} \dot{q}_{k}}{3}} .
$$

This inequality means the probability that overload occurs is less or equal to $\frac{\epsilon}{m}=\exp ^{-\beta^{2} \dot{q}_{k} / 3}$. Consequently, we can get the probability with which all vehicles satisfy the capacity constraints is at least $\left(1-\frac{\epsilon}{m}\right)^{m} \geq 1-\epsilon$. I

\section{Concluding Remarks}

In this last section, we mention some extensions to other problems and describe several open problems in this area.

The techniques derived in this paper are immediately extendible to the following problems:

1. The Hierarchical Scheduling Problems.

We derived a randomized algorithm to a simple parallel shop scheduling problem in section 3. Our techniques to derive randomized algorithms can be extended to more general environments, such as the scheduling problem with due dates, the maximum lateness minimization problem, the flow shop scheduling problem, the job-shop scheduling problem, etc. 
2. The Hierarchical Vehicle Routing Problems.

We derived a randomized algorithm to a standard vehicle routing problem in section 4. Our techniques to derive randomized algorithms can be extended to more general environments, such as the multi-depot vehicle routing problem, the time-constrained vehicle routing problem, the several problems that contain location decision, the three or more stage hierarchical vehicle routing problems, etc.

We then summarize the major open problems in the area of the hierarchical decision problems.

1. Deriving exact procedures to the hierarchical decision problems.

Using the randomized decision strategy, we can get the lower and probably good upper bounds of the hierarchical decision problems. Furthermore, if we use the a priori strategy proposed by Jaillet [23] and Bertsimas [5], the valid upper bound can be got. Combining the lower and upper bounds results in the exact or fathoming procedure to the hierarchical decision problems. This combined procedure gives more information to the decision maker than the lower bounding procedure or the upper bounding procedure alone.

2. Formulate and analyze other hierarchical decision problems in practical situations.

We showed simple examples of the hierarchical decision problems such as scheduling and vehicle routing. Many problems are remaining to be analyzed and solved.

Though the techniques suggested in this paper can be applied several other practical situations, we feel that the gaps between practice and theory are rather large; much remains to be done.

\section{Acknowledgments}

I wish to thank the anonymous referees for their valuable comments. This work was partially supported by the Waseda University Grant for Special Research Projects 63A-42 and 89A-74.

\section{References}

[1] A. V. Aho,J. E. Hopcroft and J. D. Ulman, "The Design and Analysis of Computer Algorithms," Addison-Wesley (1974).

[2] D. Angluin and L. G. Valiant, "Fast Probabilistic Algorithms for Hamiltonian Circuits and Matchings," Journal of Computing System Science, Vol. 19 (1979) pp. 155-193.

[3] O. Berman and D. Simchi-Levi," Minisum Location of a Traveling Salesman," Networks, Vol. 16 (1986) pp. 239-254.

[4] O. Berman and D. Simchi-Levi,"Finding the Optimum A Priori Tour and Location of a Traveling Salesman with Non-homogeneous Customers," Transportation Science, Vol. 22 (1988) pp. 148-154.

[5] D. Bertsimas ,"Probabilistic Combinatorial Optimization Problems," Ph. Doc. Thesis, Massachusetts Institute of Technology (1988).

[6] G. R. Bitran and A. C. Hax, "On the Design of Hierarchical Production Planning Systems," Decision Science, Vol. 8 (1977) pp. 28-55.

[7] L. Bodin and B. Golden, "Classification in Vehicle Routing and Scheduling," Networks, Vol. 11, (1981) pp. 97-108. 
[8] R. C. Burness and J. A. White ,"The Traveling Salesman Location Problem," Transportation Science, Vol. 10 (1976) pp. 348-360.

[9] A. W. Chan and R. L. Francis, "A Round Trip Location Problem on a Tree Graph," Transportation Science, Vol.10 (1976) pp.35-51.

[10] A. W. Chan and D. W. Hearn," A Rectilinear Distance Minmax Round-Trip Location Problem," Transportation Science, Vol.11 (1977) pp.107-123.

[11] A. Charnes and W. Cooper," Chance-constrained Programming," Management Science , Vol. 6 (1959) pp. 73-79.

[12] N. Christofides, A. Mingozzi , P. Toth and C. Sandi ed. ," Combinatorial Optimization: $\$ 11$ The Vehicle Routing Problem," John Wiley and Sons (1979).

[13] G. Clark and J. W. Wright," Scheduling of Vehicles from a Central Depot to a Number of Delivery Points," Operations Research, Vol. 12 (1964) pp.568-581.

[14] M. A. Dempster, M. L. Fisher, L. Jansen, B. J. Lageweg, J. K. Lenstra and A. H. G. Rinnooy Kan," Analysis of Heuristics for Stochastic Programming : Results for Hierarchical Scheduling Problems," Mathematics of Operations Research, Vol. 8 (1983) pp.525-537.

[15] C. Derman and M. Klein,"Surveillance of Multi-component Systems: A Stochastic Traveling Salesman Problem," Naval Research Logistics Quarterly, Vol.13 (1966) pp.103-111.

[16] M. Dror and P. Trudeau ," Stochastic Vehicle Routing with Modifies Saving Algorithm," European Journal Operations Research, Vol. 23 (1986) pp. 228-235.

[17] S. Eilon, C. D. T. Watson-Gandy and N. Christofides ," Distribution Management : Mathematical Modeling and Practical Analysis," Compton Printing (1971).

[18] M. Fisher and D. Hochbaum, "Probabilistic Analysis of the Planar K-Median Problem," Mathematics of Operations Research, Vol.5 (1980) pp.27-34.

[19] M. R. Garey and D. S. Johnson,"Computers and Intractability : A Guide to the Theory of NP-Completeness," Bell Telephone Labo. (1979).

[20] R. L. Graham, E. L. Lawler, J. K. Lenstra and A. H. G. Rinnooy Kan ,"Optimization and Approximation in Deterministic Sequencing ad Scheduling: A Survey," Annals of Discrete Mathematics, Vol. 5 (1979) pp.287-326.

[21] M. Haimovitch and A. Rinnooy Kan," Bounds and Heuristics for Capacitated Routing Problem," Mathematics of Operations Research, Vol.10 (1985) pp.527-542.

[22] W. Hoeffding, "On the Distribution of the Number of Successes Independent Trials," Annals of Mathematical Statistics, Vol.27 (1956) pp.713-721.

[23] P. Jaillet,"Probabilistic Traveling Salesman Problem," Ph. Doc. Thesis, Massachusetts Institute of Technology (1985).

[24] P. Jaillet and A. Odoni , "The Probabilistic Vehicle Routing Problem, Stochastic Routing Problems," in Vehicle Routing; Method and Studies, edited by B. L. Golden and A. A. Assad, Springer-Verlag, Berlin (1988) pp. 293-318.

[25] W. Jianhua,"The Theory of Games," Oxford (1988).

[26] E. L. Lawler, J. K. Lenstra, A. H. G. Rinnooy Kan and D. B. Shmoys, "The Traveling Salesman Problem," John Wiley and Sons (1985).

[27] J. K. Lenstra, A. H. G. Rinnooy Kan and L. Stougie ,"A Framework for the Probabilistic Analysis of Hierarchical Planning Systems," Annals of Operations Research, Vol. 1 (1984) pp.23-42.

[28] L. F. McGinnis ,"A Survey of Recent Results for a Class of Facility Location Problems," 
AIIE Transaction, Vol. 9 (1977) pp. 11-18.

[29] M. O'hEigeartaigh, J. K. Lenstra and A. H. G. Rinnooy Kan ed. " Combinatorial Optimization Annotated Bibliographies: $§ 6$ Probabilistic Analysis, $\$ 7$ Randomized Algorithms," (1985) John Wiley and Sons.

[30] C. H. Papadimitriou, "Worst Case and Probabilistic Analysis of a Geometric Location Problem," SIAM Journal of Computing, Vol. 10 (1981) pp. 542-557.

[31] A. Prékopa and R. J. B. Wets ed. "Stochastic Programming 84: Part I, Part II," Mathematical Programming Study, Vol. 27, 28.

[32] H. Psaraftis," On the Practical Importance of Asymptotic Optimality in Certain Heuristic Algorithms," Networks, Vol. 14 (1984) pp.587-596.

[33] M. O. Rabin ," Probabilistic Algorithms," in Algorithms and Complexity: New Directions and Recent Results, edited by J. Traub, Academic Press, New York (1976) pp. 21-39.

[34] P. Raghavan and C. D. Thompson,"Randomized Rounding: A Technique for Provably Good Algorithms and Algorithmic Proofs," Combinatorica, Vol. 7 (1987) pp. 365-374.

[35] P. Raghavan, "Probabilistic Construction of Deterministic Algorithms: Approximating Packing Integer Programs," Journal of Comp. and System Science, Vol. 37 (1988) pp. 130-143.

[36] D. Simchi-Levi and O. Berman," A Heuristic Algorithm for the Traveling Salesman Location Problem on Networks," Operations Research, Vol. 36 (1988) pp. 478-484.

[37] A. M. Spaccamela, A. H. G. Rinnooy Kan and L. Stougie," Hierarchical Vehicle Routing Problems," Networks, Vol. 14 (1984) pp. 571-586.

[38] J. M. Steel," Subadditive Euclidean Functionals and Nonlinear Growth in Geometric Probability," Annuals of Probability, Vol.9 (1981) pp.365-376.

[39] W. Stewart and B. Golden," Stochastic Vehicle Routing: A Comprehensive Approach," Euro. Jour. Oper. Res. , Vol. 14 (1983) pp. 371-385

[40] F. Tillman," The Multiple Terminal Delivery Problem with Probabilistic Demands," Transportation Science, Vol. 3 (1969) pp. 192-204.

[41] D. J. A. Welsh,"Randomized Algorithm," Discrete Applied Mathematics, Vol. 5 (1983) pp.133-145.

Mikio KUBO : Department of

Industrial Engineering and

Management,

Waseda University,

3-4-1, Okubo Shinjuku,

Tokyo 169, Japan 